\title{
The influence of pre-emptive analgesia on postoperative analgesia and its objective evaluation
}

\author{
Jitka Fricova ${ }^{1}$, Martin Vejražka², Pavel Stopka ${ }^{3}$, Jana Krizova³, Jaromír Běláček ${ }^{4}$, Richard Rokyta
}

1Department of Anaesthesiology, Resuscitation and Intensive Care, $1^{\text {st }}$ Faculty of Medicine, Charles University in Prague, Centre of Pain Management, Prague, Czech Republic

2Department of Medical Chemistry and Biochemistry, $1^{\text {st }}$ Faculty of Medicine, Charles University in Prague, Czech Republic

Institute of Inorganic Chemistry, Academy of Sciences of the Czech Republic, Řež, Czech Republic

${ }^{4}$ Department of Biophysics, $1^{\text {st }}$ Faculty of Medicine, Charles University in Prague, Czech Republic

${ }^{5}$ Department of Normal, Pathological and Clinical Physiology, $3^{\text {rd }}$ Faculty of Medicine, Charles University in Prague, Czech Republic

Submitted: 7 September 2010

Accepted: 15 September 2010

Arch Med Sci 2010; 6, 5: 764-771

DOI: 10.5114/aoms.2010.17093

Copyright $\odot 2010$ Termedia \& Banach

\section{Abstract}

Introduction: The evaluation of pain intensity is still a subject of research. Mostly psychological evaluations are used. We started to conduct biochemical evaluation in animal experiments. Now we present biochemical evaluation in postoperative pain in man.

Material and methods: In 67 patients herniotomy was done. For pre-emptive analgesia morphine and pethidine were used and the following indicators were measured: visual analogue scale (VAS), measurement of lipid spectra, saccharides and proteins, thioredoxin, super-oxide dismutase (SOD), glutathione peroxidase $(\mathrm{GPX})$ and $\mathrm{NAD}(\mathrm{P}) \mathrm{H}$-oxidase (NOX), and free radicals using electron paramagnetic resonance (EPR). Blood samples were taken and tested: before pre-medication and intervention, $4 \mathrm{~h}$ after and $24 \mathrm{~h}$ after intervention.

Results: Free radicals (FR) increased in individual samples during the postoperative course in pethidine and without pre-medication. After application of morphine the FR were insignificantly reduced. Statistically significant differences were found in albumin, prealbumin, apolipoprotein $A$, total cholesterol, atherosclerotic index, CRP, glucose, and thioredoxin $(p \leq 0.001)$. A greater difference was seen in VAS values between morphine and pethidine premedications $(p \leq 0.001)$.

Conclusions: It was proved that the biochemical markers of lipid, protein and saccharide metabolisms and free radicals as well as singlet oxygen can serve as very good indicators of the intensity of pain and nociception. In patients it was proved that pre-emptive analgesia plays an important role in reducing the intensity of postoperative pain. From the three modalities of pre-emptive analgesia morphine represents the best solution.

Key words: postoperative pain, objective evaluation, preemptive analgesia, morphin, pethidin, VAS.

\section{Introduction}

Acute postoperative pain is perceived by the organism as a strong stimulus which evokes and consequently triggers reactions such as

\author{
Corresponding author: \\ Jitka Fricová, MD \\ Department of \\ Anaesthesiology, Resuscitation \\ and Intensive Care \\ $1^{\text {st }}$ Faculty of Medicine \\ Charles University in Prague \\ Centre of Pain Management \\ Karlovo náměstí 32 \\ 128 00, Praha 2 \\ Czech Republic \\ E-mail: j.fricova@seznam.cz
}


nociception, sensitization (peripheral or central) and neurohumoral modulation. Some co-authors of this paper, in previous studies, in which they monitored biochemical parameters during pain, concentrated their attention on changes in lipid, protein and saccharide parameters associated with chronic pain [1].

Studies on patients with bone fractures and acute pancreatitis have monitored similar parameters [1]. The most significant changes were described in the lipid spectrum, especially LDL and $\mathrm{HDL}$ cholesterol. Changes in LDL cholesterol were significantly higher than those found in healthy patients. HDL cholesterol levels were significantly higher, especially in patients with acute pancreatitis, compared to healthy patients and patients with bone fractures. LDL cholesterol returned to original values 30 days after pain onset in both groups with pain. The "on admission" visual analogue scale (VAS) was higher in pancreatitis patients than in patients with fractures.

These studies demonstrate that biochemical changes in blood are measurable during acute and chronic pain. The question remains whether these biochemical correlates can be regarded as common markers. In light of the fact that the more remarkable changes were confirmed in acute intensive pain caused by fractures and pancreatitis we chose acute postoperative pain as the next topic of interest in our continuing study of oxidative stress.

It was discovered in 1913 that acute postoperative pain can be intensified and fixed in a state of neural hyperexcitability as a result of a surgical intervention. Many years later Wall [2] proposed that pre-emptive preoperative analgesia can block central neuronal sensitization and reduce acute postoperative pain.

The hypothesis that surgical incisions act as central sensitization triggers began to be slowly accepted and integrated into other views regarding postoperative pain; i.e. sensitizing effects include both preoperative and postoperative injurious stimuli. Up to now, it has not been evident whether general anaesthesia is able to suppress or block the transmission of nociception from the periphery to the spinal cord and brain [4]. Additionally, systematically applied opioids cannot be considered as sufficient prevention against central sensitization [5].

There have been many randomized, double blind clinical studies designed to prove or disprove the benefit of pre-emptive analgesia. Katz and McCartney [6] studied a group of 175 patients in a controlled, double blind study. They evaluated, with regard to pre-emptive analgesia, the influence of local anaesthetics, opioids, NSAIDs, NMDA agonists, and clonidine (alone and in combination with opioids and multimodal analgesia). They also evaluated the local influence of anaesthetics before tonsillectomy and before herniotomy. In recent years, oxidative stress has been found to play a crucial role in the pathophysiology of several diseases.

The objectives of this study were: to compare biochemical changes which originate after preemptive analgesia and after surgical intervention; and to establish which of three combinations of commonly used, pre-emptive analgesia is the most effective from the point of view of postoperative pain and oxidative stress.

For evaluation of pain intensity the following methods were used: VAS, biochemical evaluation of lipid, saccharide and protein metabolism, free radical measurements.

\section{Material and methods}

\section{Patients}

The study involved patients after surgical interventions ( $n=67$ ) with an expected pain intensity on the VAS (1-10) greater than 5 . The group was composed of 55 men and 12 women. The surgical intervention that served as the model of acute postoperative pain was a herniotomy of a hernia inguinalis. The control group was created from patients receiving routine analgesia (pethidine).

On the basis of a pilot study, we decided to monitor the main analgesics used for premedication before surgical intervention such as strong opioids (morphine and pethidine) and compare the results with a group of patients without premedication.

\section{Evaluation of pain intensity}

We used subjective and objective methods for evaluation of pain intensity and its objectification. 1. Subjective evaluation: VAS.

2. Objective evaluation:

a. measurement of lipid spectra, saccharides and proteins;

b. measurement of oxidative stress parameters - thioredoxin in serum, activity of super-oxide dismutase (SOD), glutathione peroxidase (GPx) and NAD(P)H-oxidase (NOX); and

c. measurement of free radicals using electron paramagnetic resonance (EPR).

Blood samples were taken and tested on the following time scale:

a. before premedication and intervention;

b. $4 \mathrm{~h}$ after intervention; and

c. $24 \mathrm{~h}$ after intervention.

Pain intensity was evaluated (VAS) at the same times as blood samples were taken.

\section{Electron paramagnetic resonance methodology}

Electron paramagnetic (spin) resonance spectroscopy uses the interaction between 
magnetic fields and microwave energy to identify and determine free radicals, paramagnetic complexes and excited states. A free radical is a particle with one or more unpaired electrons and is generally highly reactive. The method of "spin capture" is used for the study of short-lived radicals (nanoseconds). Within this context, chemicals (spin traps) are used which, together with free radicals, create a product with a longer life-span. Usually this technique is used to study hydroxyl $(\mathrm{OH})$, super oxide $\left(\mathrm{O}_{2}^{-}\right)$, nitroxide (NO) and peroxy radicals (ROO).

The method used in the current experiment has been described and refined over the years and is well documented in the literature [7-9]. The results from these previous studies gave us a baseline of previously characterized spectra of radicals which allowed identification and determination of concentrations of radical in our samples.

\section{Measurement and evaluation of samples}

A spin trap, DMPO (5,5,-dimethyl-pyrroline $\mathrm{N}$-oxide, Sigma-Aldrich) or PBN (phenyl-tertbutylnitrone), was immediately added to the samples and the samples were then frozen in liquid nitrogen and transported.

The acquired spectra were evaluated using the Bruker-Biospin program and visualized using the graphics program Origin. Bruker-Biospin made it possible to directly read spectral constants (g-factor, signal amplitudes $[\Delta \mathrm{Hpp}$, in magnetic field units, gauss, or millitesla]), location of signals in the magnetic field, and signal intensity (App, in work units EPR). The signals were compared with spectra standards $\left(\mathrm{Mn}^{2+} / \mathrm{ZnS}, \mathrm{Cr}^{3+} / \mathrm{MgO}\right.$, Magnettech, Berlin, Germany). Singlet oxygen was studied after application of 2,2,6,6-tetramethylpiperidine (TMP), which is specific for singlet oxygen $\mathrm{O}_{2}^{-}$determination. This compound selectively reacts with singlet oxygen, creating relatively stable nitroxide radicals. The triplet spectrum corresponds to the quantity of singlet oxygen.

We measured the following enzymes associated with free radicals:

Superoxide dismutase is one of the most important enzymes in the metabolism of reactive oxygen species (ROS). It catalyses the conversion of superoxide to hydrogen peroxide. The activity of superoxide dismutase (SOD) is measured spectrophotometrically.

Glutathione has recently been considered as the most important scavenger of hydrogen peroxide in most tissues. During oxidative stress, GPx activity is increased as stress increases.

NADPH oxidase is today regarded as one of the leading producers of ROS in vascular tissue. It is involved both in the regulation of certain physiological functions of blood vessels (e.g. regulation of blood pressure, permeability, angioneogenesis) and in the development of several diseases (hypertension, atherosclerosis, hypertrophy of the media in cardiovascular disease, angioneogenesis, tumours, etc). As a part of the thioredoxin system, and according to new studies, it has a key role in removing hydrogen peroxide. During oxidative stress, thioredoxin is the first to show a sharp increase in plasma concentrations. It is measured immunochemically using the ELISA test.

\section{Statistical analysis}

Statistical analysis of results was performed using the SPSS GLM procedure (General Linear Model for Repeated Measures) and by NPAR TESTS (Kolmogorov-Smirnov test to verify the standard normality assumptions and Kruskal-Wallis and Friedman tests when normality assumptions did not hold for any input indicators). The usual posthoc analysis method, LSD (least significant difference) t-tests and pair comparison due to REPEATED contrast type, was performed for situations in which the null hypothesis was rejected because of the homogeneous influence of premedication ( 1 - pethidine, 2 - morphine, 3 - without anaesthesia) and regarding the influence of time factors at the $1^{\text {st }}, 2^{\text {nd }}$ and $3^{\text {rd }}$ measurement recomputed towards the $1^{\text {st }}$ one taken as the base on levels of all experimental indicators (excluding VAS and free radicals, where we used the original values for statistical evaluations). All statistical tests were compared with critical $p$-values of $p=0.05$ or lower.

\section{Results}

The results of measurements of biochemical changes in patients with different types of premedication are shown in Table I.

Measurement of free radicals using EPR (Figure 1). Free radicals (FR) increased in individual samples during the postoperative course in pethidine and without premedication. After application of morphine the FR were insignificantly reduced, especially in the third sampling. The increase in measured values of free radicals is statistically significant in patients without premedication between the $2^{\text {nd }}$ and $3^{\text {rd }}$ collections.

\section{Measurement of biochemical parameters}

Total protein - no statistically significant results. Albumin (Figure 2) - statistically significant lower levels of albumin in patients "before" the operation for all pre-treatments $(p<0.001)$; it is most pronounced for morphine, less for pethidine and patients without premedication.

Prealbumin (Figure 3) - indices for prealbumin have the opposite trend to that of albumin. 
Table I. Average (mean) values and SEM for before (1) and after (2 and 3) parameters for total proteins, prealbumin, apolipoprotein A, apolipoprotein B, triacylglycerols, cholesterol (total, HDL, LDL), CRP and uric acid in the framework of each individual measurement converted to level of indicator albumin

\begin{tabular}{|c|c|c|c|c|c|c|c|c|}
\hline \multirow[t]{2}{*}{ Premedication } & \multirow[t]{2}{*}{ Variable } & \multirow[t]{2}{*}{$\mathrm{N}$} & \multicolumn{2}{|c|}{ 1. Before premedication } & \multicolumn{2}{|c|}{ 2. After premedication } & \multicolumn{2}{|c|}{ 3. After surgery } \\
\hline & & & Mean & SEM & Mean & SEM & Mean & SEM \\
\hline \multirow{14}{*}{$\begin{array}{l}\text { Without } \\
\text { premedication }\end{array}$} & Total proteins & 18 & 69.88 & 1.46 & 63.69 & 0.85 & 66.57 & 0.99 \\
\hline & Albumin & 18 & 42.88 & 1.08 & 38.89 & 0.45 & 40.62 & 0.61 \\
\hline & Prealbumin & 16 & 0.27 & 0.02 & 0.24 & 0.02 & 0.23 & 0.02 \\
\hline & HDL cholesterol & 16 & 1.23 & 0.07 & 1.07 & 0.06 & 1.11 & 0.06 \\
\hline & LDL cholesterol & 15 & 2.92 & 0.20 & 2.74 & 0.14 & 2.65 & 0.17 \\
\hline & Triacylglycerols & 16 & 1.63 & 0.29 & 1.42 & 0.25 & 1.39 & 0.21 \\
\hline & Glucose & 15 & 5.62 & 0.36 & 5.18 & 0.31 & 5.96 & 0.46 \\
\hline & Uric acid & 6 & 345.33 & 19.06 & 329.33 & 16.23 & 355.00 & 14.17 \\
\hline & CRP & 16 & 7.76 & 3.70 & 7.84 & 3.30 & 39.91 & 7.07 \\
\hline & SOD & 9 & 2.06 & 0.65 & 2.54 & 0.84 & 2.37 & 0.93 \\
\hline & GPX & 12 & 1.20 & 0.24 & 0.93 & 0.16 & 1.21 & 0.20 \\
\hline & NOX nonstim & 21 & 0.88 & 0.38 & 0.65 & 0.50 & 0.84 & 0.66 \\
\hline & Free radicals & 22 & 3.04 & 0.44 & 3.06 & 0.25 & 4.06 & 0.44 \\
\hline & VAS & 24 & 2.13 & 0.36 & & & 5.71 & 0.35 \\
\hline \multirow[t]{14}{*}{ Pethidine } & Total proteins & 14 & 72.37 & 1.93 & 63.68 & 2.29 & 66.53 & 2.24 \\
\hline & Albumin & 14 & 45.86 & 0.92 & 41.83 & 0.71 & 43.31 & 0.69 \\
\hline & Prealbumin & 12 & 0.27 & 0.02 & 0.24 & 0.02 & 0.23 & 0.02 \\
\hline & HDL cholesterol & 13 & 1.29 & 0.09 & 1.10 & 0.06 & 1.15 & 0.07 \\
\hline & LDL cholesterol & 12 & 2.91 & 0.26 & 2.65 & 0.25 & 2.83 & 0.29 \\
\hline & Triacylglycerols & 13 & 1.84 & 0.42 & 1.65 & 0.43 & 1.45 & 0.37 \\
\hline & Glucose & 14 & 4.76 & 0.19 & 4.65 & 0.21 & 5.29 & 0.24 \\
\hline & Uric acid & 10 & 323.30 & 11.78 & 314.70 & 13.45 & 346.40 & 20.87 \\
\hline & CRP & 14 & 5.51 & 1.43 & 7.14 & 2.73 & 36.09 & 7.28 \\
\hline & SOD & 17 & 13.17 & 5.05 & 15.45 & 6.58 & 12.97 & 4.91 \\
\hline & GPX & 19 & 0.78 & 0.19 & 0.84 & 0.18 & 0.86 & 0.23 \\
\hline & NOX nonstim & 20 & 1.18 & 0.28 & 0.62 & 0.35 & 0.28 & 0.37 \\
\hline & Free radicals & 26 & 2.61 & 0.31 & 3.17 & 0.33 & 3.49 & 0.37 \\
\hline & VAS & 26 & 3.12 & 0.33 & & & 5.12 & 0.34 \\
\hline \multirow[t]{14}{*}{ Morphine } & Total proteins & 13 & 71.85 & 1.07 & 65.8 & 1.57 & 69.51 & 1.49 \\
\hline & Albumin & 13 & 44.18 & 0.55 & 40.51 & 0.73 & 42.60 & 0.72 \\
\hline & Prealbumin & 11 & 0.27 & 0.02 & 0.25 & 0.02 & 0.23 & 0.02 \\
\hline & HDL cholesterol & 13 & 1.13 & 0.08 & 0.99 & 0.06 & 1.08 & 0.06 \\
\hline & LDL cholesterol & 13 & 3.16 & 0.30 & 3.03 & 0.29 & 3.05 & 0.32 \\
\hline & Triacylglycerols & 13 & 1.58 & 0.18 & 1.31 & 0.16 & 1.20 & 0.10 \\
\hline & Glucose & 13 & 5.49 & 0.50 & 5.40 & 0.47 & 5.92 & 0.50 \\
\hline & Uric acid & 13 & 366.77 & 17.16 & 361.77 & 21.31 & 372.15 & 21.05 \\
\hline & CRP & 11 & 7.00 & 3.45 & 8.00 & 4.82 & 43.87 & 10.06 \\
\hline & SOD & 13 & 1.18 & 0.20 & 1.11 & 0.12 & 1.10 & 0.17 \\
\hline & GPX & 16 & 0.77 & 0.16 & 0.66 & 0.14 & 1.05 & 0.13 \\
\hline & NOX nonstim & 16 & 1.16 & 0.27 & -0.12 & 0.31 & -0.04 & 0.28 \\
\hline & Free radicals & 17 & 3.69 & 0.32 & 3.66 & 0.23 & 3.46 & 0.31 \\
\hline & VAS & 17 & 3.71 & 0.32 & & & 6.41 & 0.17 \\
\hline
\end{tabular}


Free Rad *Premedication LS Means Vertical bars denote \pm standard errors

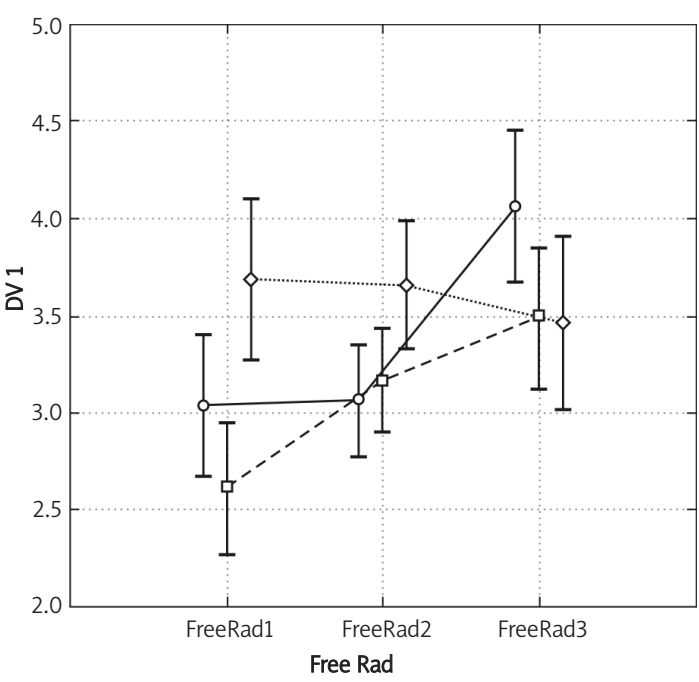

푸으 Premedication 1 without premedication

-도- Premedication 2 pethidin

…… Premedication 3 morphin

Figure 1. The levels of free radicals measured before surgery, $4 \mathrm{~h}$ after surgery and $24 \mathrm{~h}$ after surgery in patients divided into 3 groups according to pretreatment by Pethidine, Morphine and without premedication

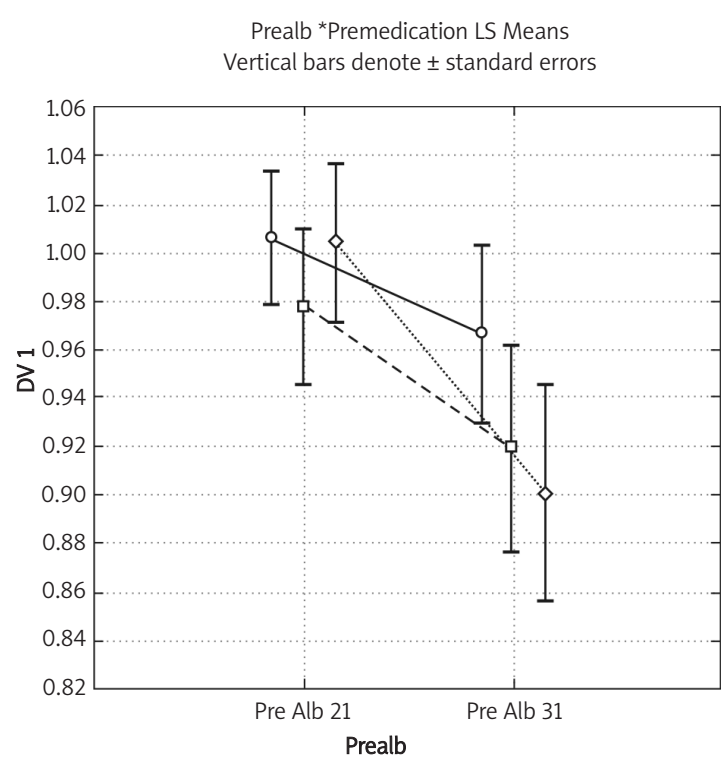

్ㅜㄷㅡ Premedication 1 without premedication

-도- Premedication 2 pethidin

$\cdots \bar{\ldots} \ldots$ Premedication 3 morphin

Figure 3. The levels of prealbumine measured before surgery, $4 \mathrm{~h}$ after surgery and $24 \mathrm{~h}$ after surgery in patients divided into 3 groups according to pretreatment by Pethidine, Morphine and without premedication
Albumine *Premedication LS Means Vertical bars denote \pm standard errors

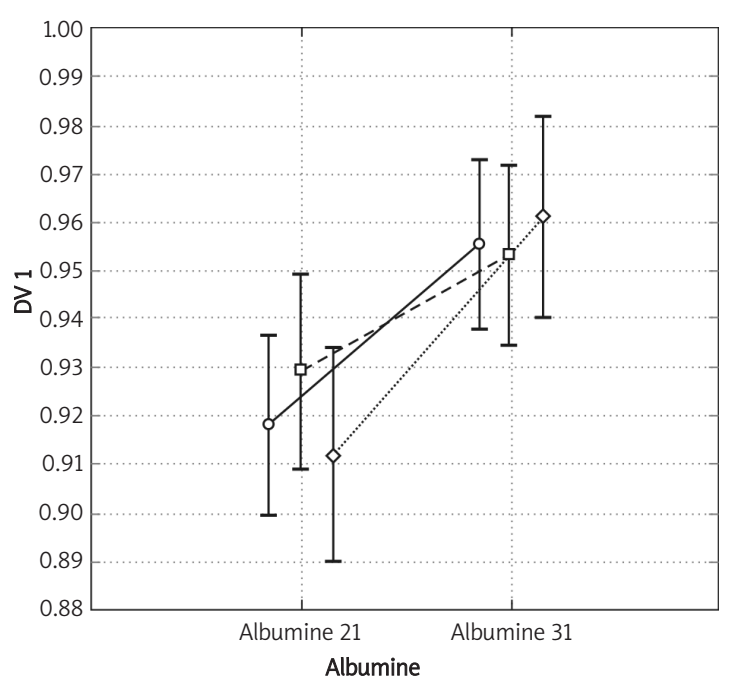

푸으 Premedication 1 Without Premedication

-亭 - Premedication 2 Pethidin

$\overline{\cdots .} \bar{\ldots}$ Premedication 3 Morphin

Figure 2. The levels of albumine measured before surgery, $4 \mathrm{~h}$ after surgery and $24 \mathrm{~h}$ after surgery in patients divided into 3 groups according to pretreatment by Pethidine, Morphine and without premedication

A statistically significant difference, however, was only present for morphine $(p=0.007)$.

Apolipoprotein A did not show a statistically significant decrease.

Apolipoprotein B showed a statistically significant decrease "after" surgery only for patients who received morphine ( $p=0.021$ for Friedman's test). Patients "after" surgery had significantly higher values relative to pethidine and lower relative to morphine ( $p=0.033$ for Kruskal-Wallis test).

Triacylglycerols (TAG) - no statistically significant results.

Total cholesterol levels decreased for all three premedications between the first and second sampling. The decrease in index values "after" surgery was statistically significant for patients without premedication $(p<0.046)$ and also when morphine was used as the premedication $(p=0.004)$. Total cholesterol "before" surgery was significantly higher in patients without premedications and lower in patients premedicated with morphine ( $p=0.049$ using ANOVA).

$\mathrm{HDL}$ cholesterol - no statistically significant results.

Atherosclerotic index - index values for patients "after" surgery were significantly lower for all patients $(P<0.027)$.

CRP values "after" surgery were significantly higher than "before" surgery. Changes for all pretreatments were significant $(p<0.002)$. 
Glucose - the increase in glucose "after" surgery was statistically significant for pethidine pretreatment $(p=0.001)$.

Uric acid - the level of uric acid did not significantly change.

Thioredoxin - values "before" and "after" surgery are significantly different, especially when compared to those without premedication and those premeditated with pethidine or morphine.

VAS - the "before" surgery and "after" surgery values were statistically different $(p<0.001)$. A greater difference was seen $(p<0.001)$ regarding the VAS values in the case of morphine premedication compared with pethidine premedication.

\section{Discussion}

Our goal was to determine whether pre-emptive analgesia had a positive effect on acute postoperative pain. Assuming there was a positive effect, we next wanted to know which types of analgesics were associated with the best preemptive and postoperative analgesia. Finally, we wanted to determine how acute pain affects the patient and whether it is possible to measure and describe these changes.

We have studied postoperative analgesia effects involving different types of preoperative and postoperative medication and its objective evaluation. We concentrated on objective pain evaluation using different biochemical parameters, especially free radicals, which are significantly changed during acute pain. Besides finding the optimal pre-emptive and postoperative analgesia, our target was to explain the common mechanisms of postoperative pain and oxidative stress. Our goal was the objectification of biochemical changes based on acute postoperative stress.

The objective evaluation of pain intensity is a long-term problem, because the objectification using electrophysiological and visual methods is, at present, demanding and expensive, and therefore [10], in laboratories in the Czech Republic and many other places as well, the evaluation is performed using biochemical methods.

We implemented a large experimental study using mechanical and thermal models of nociception and we discovered that, in particular, hydroxyl free radicals, nitroxide free radicals and singlet oxygen were significantly increased after painful nociceptive stimulation. This increase can be suppressed by antioxidants, and simultaneously it was shown that some parameters of lipid, saccharide, and protein metabolism were increased [11-13]. These experimental results were partially verified using different types of acute and chronic pain in humans (acute pancreatitis, fractures, cholecystitis, pain of visceral origin and low-back pain $[1,7]$. All these painful syndromes reflected different biochemical parameters.

Reducing pain intensity following all types of interventions is an important clinical goal. Our goal was to find the most effective analgesic combination for effective treatment of acute postoperative pain.

The experimental studies performed on rats paid attention to oxidative stress studied after repeated painful stimuli [11-13]. Unlike the present study in patients, we used different analgesics such as morphine, as well as combinations of drugs.

The MDA and antioxidant changes were probably specific for each pain mechanism.

Another study [12] involved the direct measurement of free radicals in the brain and in the blood after painful stimulation (rats).

An important oxidant was found to be thioredoxin reductase [14]. In recent years, oxidative stress has been found to play a crucial role in the pathophysiology of cardiovascular diseases such as essential hypertension and rhythm-related disorders.

Proteins, as a result of their abundance in biological systems, are major targets for oxidants, and they have high reaction rate constants. Kinetic data for a number of radicals and non-radical oxidants (e.g. singlet oxygen and hypochlorous acid) are consistent with proteins consuming the majority of these species generated within cells. Most protein damage is non-repairable and has deleterious consequences for protein structure and function; methionine sulfoxide formation can however be reversed in some circumstances. The major fate of oxidized proteins is catabolism by proteasomal and lysosomal pathways, but some materials appear to be poorly degraded and accumulate within cells. The accumulation of damaged material may contribute to a range of human pathologies. It seems that elevated methionine plays a key role in a very large number of human and animal diseases [15].

Postoperative adhesion formation has been associated with the occurrence of increased levels of ROS [16, 17]. The production of ROS is an important part of the immune defence, and ROS are normally quickly detoxified by biological antioxidant systems. However, when the amount and activity of ROS increase beyond the power of antioxidant systems, they cause deleterious oxidative stress in tissues [18]. Reactive oxygen species are produced in hyperoxic environments and have, accordingly, been found to increase during open surgery where externalized tissues are exposed to atmospheric oxygen [19]. In air $\mathrm{O}_{2}$ has a partial pressure of $160 \mathrm{mmHg}(21 \%$ of $760 \mathrm{mmHg}$ ), which is much higher than the intracellular $\mathrm{O}_{2}$ pressures. Consequently, conditions during open surgery will inevitably lead to local 
hyperoxia and increased occurrence of ROS and oxidative stress. Laparoscopic surgery, on the other hand, does not seem to cause oxidative stress [19]. This advantage has been ascribed to the avoidance of air exposure by intraperitoneal insufflation with $\mathrm{CO}_{2}[19]$. Therefore, we correspondingly suggest that intraoperative field flooding with $\mathrm{CO}_{2}$ may reduce oxidative stress and thus the risk of adhesion formation associated with open surgery, simply because the resulting local $\mathrm{CO}_{2}$ atmosphere will exclude ambient air and the reactive $\mathrm{O}_{2}$ which is present in it $[18,20]$.

During open surgery, an increase in ROS production has been reported, i.e. an increase in superoxide anions, xanthine oxidase, an enzyme involved in ROS formation [21], and malondialdehyde (MDA), a marker of ROS production [22]. ROS also increase during laparoscopic surgery. Indeed, recent findings showed an increase of markers of ROS production such as 8-iso-prostaglandin F2 and hydroxyeicosatetraenoic acid in human peritoneum in a time- and $\mathrm{CO}_{2}$ volume-dependent manner [22] and of malondialdehyde in the intestine, liver and lung in rats [23]. In addition, a negative correlation between ROS scavengers such as GHS-Px, SOD, CAT and $\mathrm{GSH}$, and the duration/amount of $\mathrm{CO}_{2}$ exposure was observed [24].

Morphine and ROS: one study examined whether intracellular ROS was generated in cultured human umbilical vein endothelial cells treated with morphine. Use of naloxone, to analyse the rescue effect, showed that cultures of human umbilical vein endothelial cells (pre-treated with either low (102 nM) or high (104 nM) dosage of naloxone could reverse morphine-mediated ROS production. In recent papers morphine was also studied in domestic animals (Bos taurus and Equus caballus) and it was found that the hormones and neurotransmitters release it in different tissues [25]. In contrast, pethidine is not a suitable anaesthetic for open cholecystectomy with regard to postoperative care [26].

The results showed that caspases 3 and 7 were involved in morphine-induced apoptosis in cultures of human umbilical vein endothelial cells and opioid receptors were important in morphine-mediated caspase production [27].

It was found that the biochemical markers of lipid, protein and saccharide metabolism and free radicals as well as singlet oxygen can serve as very good indicators of the intensity of pain and nociception.

In the cohort of patients with postoperative pain after laparotomy for hernia inguinalis it was found that pre-emptive analgesia plays an important role in reducing the intensity of postoperative pain.

From three modalities of pre-emptive analgesia (without premedication, premedication with pethidine, premedication with morphine) we obtained the best influence of postoperative pain after pre-emptive analgesia with morphine.

\section{Acknowledgements}

The work was supported by Research Goal MSM 0021620816.

\section{References}

1. Krrikava K, Kalla K, Yamamotová A, Rokyta R. Blood serum changes in patients with pain during bone fractures and acute pancreatitis. Neuro Endocrinol Lett 2004; 25: 62-9.

2. Wall PD. The prevention of postoperative pain. Pain 1988; 33: 289-90.

3. Wall PD, Melzack R, editors. Textbook of pain. $4^{\text {th }}$ ed. Edinburgh: Churchill Livingston; 1999, 165-81.

4. Rundshagen I, Kochs E, Schulte am Esch J. Surgical stimulation increases median nerve somatosensory evoked responses during isoflurane-nitrous oxide anaesthesia. Br J Anaesth 1995; 75: 598-602.

5. Abram SE, Yaksh TL. Morphine, but not inhalation anesthesia, blocks post-injury facilitation. The role of preemptive suppression of afferent transmission. Anesthesiology 1993; 78: 713-21.

6. Katz J, McCartney CJ. Current status of preemptive analgesia. Curr Opin Anaesthesiol 2002; 15: 435-41.

7. Rokyta R, Stopka P, Kafunkova E, Krizova J, Fricova J, Holecek $V$. The evaluation of nociceptive intensity by using free radicals direct measurement by EPR method in the tail of anaesthetized rats. Neuro Endocrinol Lett 2008; 29: 1007-14.

8. Gallez B, Schwartz HM. In vivo EPR: when, how and why? NMR Biomed 2004; 17: 223-5.

9. Štípek S, editor. Antioxidanty a volné radikály ve zdraví a v nemoci. 1 $1^{\text {st }}$ ed. Praha: Grada, 2000.

10. Mao Y, Zang L, Shi X. Singlet oxygen generation in the superoxide reaction. Biochem Mol Biol Int 1995; 36: 227-32.

11. Rokyta R, Holeček V, Pekárková I, et al. Free radicals after painful stimulation are influenced by antioxidants and analgesics. Neuro Endocrinol Lett 2003; 24: 304-9.

12. Rokyta R, Stopka P, Holeček, Krìikava K, Pekárková I. Direct measurement of free radicals in the brain cortex and the blood serum after nociceptive stimulation. Neuro Endocrinol Lett 2004; 25: 252-6.

13. Fricova J, Stopka P, Krizova J, Yamamotova A, Rokyta R. The effect of laparotomy on hydroxyl radicals, singlet oxygen and antioxidants measured by EPR method in the tails of rats. Neuro Endocrinol Letters 2009; 30: 373-6.

14. Holecek V, Liska J, Racek J, Rokyta R. The significance of free radicals and antioxidants due to the load induced by sport activity [Czech]. Cesk Fysiol 2004; 53: 76-9.

15. Davies MJ. The oxidative environment and protein damage. Biochim Biophys Acta 2005; 17: 93-109.

16. Binda MM, Molinas CR, Koninckx PR. Reactive oxygen species and adhesion formation clinical implications in adhesion prevention. Hum Reprod 2003; 18: 503-7.

17. Elkelani OA, Binda MM, Molinas CR, Koninckx PR. Effect of adding more than $3 \%$ oxygen to carbon dioxide pneumoperitoneum on adhesion formation in a laparoscopic mouse model. Fertil Steril 2004; 82: 1616-22.

18. Persson $M$, van der Linden J. Intraoperative field flooding with warm humidified $\mathrm{CO} 2$ may help to prevent adhesion formation after open surger. Med Hypotheses 2009; 73: 521-3. 
19. Tsuchiya M, Sato EF, Inoue M, Asada A. Open abdominal surgery increases intraoperative oxidative stress: can it be prevented? Anesth Analg 2008; 107: 1946-52.

20. Persson $M$, Svenarud $P$, van der Linden J. What is the optimal device for carbon dioxide deairing of the cardiothoracic wound and how should it be positioned? J Cardiothorac Vasc Anesth 2004; 18: 180-4.

21. Anup R, Aparna V, Pulimood A, Balasubramanian KA. Surgical stress and the small intestine: role of oxygen free radicals. Surgery 1999; 125: 560-9.

22. Souza AMB, Rogers MS, Wang CC, Yuen PM, Ng PS. Comparison of peritoneal oxidative stress during laparoscopy and laparotomy. J Am Assoc Gynecol Laparosc 2003; 10: 65-74.

23. Eleftheriadis E, Kotzampassi K, Botsios D, Tzartinoglou E, Farmakis H, Dadoukis J. Splanchnic ischemia during laparoscopic cholecystectomy. Surg Endosc 1996; 10: 324-6.

24. Taskin O, Sadik S, Onoglu A, et al. Adhesion formation after microlaparoscopic and laparoscopic ovarian coagulation for polycystic ovary disease. J Am Assoc Gynecol Laparosc 1999; 6: 159-63.

25. Mantione KJ. Morphine signaling in Bos taurus and Equus caballus. Arch Med Sci 2009; 5: 613-7.

26. Raddi P, Nagalingaswamy VP, Khatib F, Wang Y, Chandra SBC. A comparison of interpleural bupivacaine and intravenous pethidine for postoperative pain relief following open cholecystectomy. Arch Med Sci 2009; 5, 1: 57-62

27. Hsiao PN, Chang MC, Cheng WF, et al. Morphine induces apoptosis of human endothelial cells through nitric oxide and reactive oxygen species pathways. Toxicology 2009; 4: 83-91 